\title{
The supreme turbinate and the drainage of the posterior ethmoids: a computed tomographic study
}

\author{
T. Gotlib1 , M. Kuźmińska1 , J. Sokołowski ${ }^{1}$, T. Dziedzic², K. Niemczyk1 \\ ${ }^{1}$ Department of Otolaryngology, Medical University of Warsaw, Poland \\ ${ }^{2}$ Department of Neurosurgery, Medical University of Warsaw, Poland
}

[Received: 23 January 2017; Accepted: 2 April 2017]

\begin{abstract}
Background: It is generally acknowledged that the posterior ethmoidal cells drain under the superior nasal turbinate (SorNT) or, rarely, under the supreme nasal turbinate (SmeNT), and the sphenoid ostium (SO) opens to the sphenoethmoidal recess. However, detailed relations between these structures are variable, complex and still not clear. There is no reliable data on the prevalence of SmeNT and drainage of the posterior ethmoidal cells under this structure. The aim of this study was to re-evaluate the anatomy of the aforementioned region.

Materials and methods: Multiplanar and three-dimensional reconstruction analysis of 100 thin slice paranasal sinus computed tomography scans.

Results: SmeNT was identified in 77 subjects (136 sides). It formed the ostium to the posterior ethmoidal cell adjacent to the skull base or orbit in 58 subjects (91 sides). This cell drained independently from the remaining posterior ethmoidal cells. The sphenoethmoidal (Onodi) cell drained to supreme meatus in 41 subjects ( 54 sides), and to superior meatus in 37 subjects (49 sides). SO was always located medial to the posteroinferior attachment of SmeNT, or SorNT (in absence of SmeNT).

Conclusions: Patients with divergent drainage of the posterior ethmoids (with posterior ethmoidal cell draining to the supreme meatus) may require more extensive surgery to avoid persistence or recurrence of inflammatory disease. SmeNT is more common than thought, but due to its posterior and superior location to SorNT, it is rarely seen intraoperatively. If SmeNT is present, SO is always located medial to its posteroinferior attachment. (Folia Morphol 2018; 77, 1: 110-115)
\end{abstract}

Key words: turbinates, anatomy, computerised tomography, ethmoid sinus, sphenoid sinus, drainage

\section{INTRODUCTION}

The supreme nasal turbinate (SmeNT) is the smallest of all nasal turbinates. Its prevalence varies between $8 \%$ and $50 \%$ according to different authors [7-9] SmeNT has been outside the scope of interest of otorhinolaryngologists and skull base surgeons. It is rather ignored as an insignificant anatomical oddity. The opening of the posterior ethmoidal cell into the supreme meatus has been cursorily mentioned in anatomical studies and textbooks $[5,9]$ but it is not a constant feature of SmeNT [9]. SmeNT potentially divides two distinct areas of drainage, similarly to

Address for correspondence: M. Kuźmińska MD, Department of Otolaryngology, Medical University of Warsaw, ul. Banacha 1a, 02-097 Warszawa, Poland, tel: +48 22 5991021, fax: +48 22599 2523, e-mail: magda.kuzminska@wp.pl 
the middle turbinate. Thus the knowledge of detailed anatomy of SmeNT and related posterior ethmoidal cells may improve understanding of pathophysiology of chronic rhinosinusitis and influence surgical decision making. To our knowledge the prevalence and morphology of the posterior ethmoidal cells draining under SmeNT has not been evaluated so far.

There is a paucity of data in the literature on the prevalence and anatomy of SmeNT and its relation to superior nasal turbinate (SorNT) and the sphenoid ostium (SO). Most of the studies on this topic were based on limited number of cadavers. Results of these studies are not consistent [7-9].

Superior nasal turbinate is regarded as a reliable anatomical landmark for the identification of so during transnasal and transethmoidal approach to the sphenoid sinus $[1,10]$. It is generally accepted that SO is located medial to SorNT in most cases; however, there are conflicting data on the prevalence of SO located laterally to SorNT $[6,8]$. Coming across SmeNT during surgery may lead to confusion and lack of confidence regarding the location of SO [2].

Currently available three-dimensional computed tomography (3D CT) reconstruction software gives the possibility to visualise the lateral nasal wall as it looks in a living individual. Unlike in the anatomical specimen, a wrong cut can be rectified during virtual dissection.

The aim of this study was to describe the prevalence and morphology of SmeNT and its relation to SorNT and SO, and to evaluate the posterior ethmoidal cells draining under SmeNT.

\section{MATERIALS AND METHODS}

The study was approved by the University Ethics Committee. Overall $1850 \mathrm{CT}$ examinations performed due to sinonasal complaints were retrospectively assessed. The following were excluded from the study: examinations acquired with slice thickness greater than $0.67 \mathrm{~mm}$, with gantry tilt, showing any opacification of the posterior ethmoid cells, sphenoid sinus, and after surgery of the posterior ethmoids or sphenoidal sinus. Following exclusion based on the aforementioned criteria, those scans which showed gross septal deviation were eliminated. The remaining 100 examinations of adult Caucasians (61 females and 39 males) mean age 44 years (standard deviation $[S D]=17$, range 19-83 years) were evaluated further using multiplanar reconstruction (MPR) and 3D reconstruction with Osirix (Pixemo, Switzerland) software. The coronal, horizontal and sagittal planes were set using lateral aspect of orbits, the floor of the nasal cavity and crista galli. The images were viewed in established planes (as described above) and with the anterior and lateral tilt. MPR images were analysed in the bony window (window level: 300; window width: 1500), at slice thickness from $0.1 \mathrm{~mm}$ to $0.25 \mathrm{~mm}$ (Fig. 1). Convex longitudinal structure located above SorNT, greater than $3 \mathrm{~mm}$ in size, with a separate posterior attachment was classified as SmeNT. The posterior ethmoidal cells draining below SmeNT were evaluated for their relation to the ethmoid roof and the optic nerve.

\section{Three-dimensional reconstruction}

Three-dimensional head reconstruction was created using a "3D rendering" tool. In this image reconstruction technique, different colours and transparencies are assigned to different intensity values in $\mathrm{CT}$ dataset. The alignment of cutting planes at different angles, and the virtual removal of selected volumes together with adjustments of contrast and virtual shading make it possible to create clear 3D images. The structures covering the posterior lateral wall of the nasal cavity were virtually removed, and the SO was visualised. The same procedure was repeated on both sides (Fig. 1).

The views of the left and right lateral wall of the nasal cavity were saved for further analysis. Basing on the lateral 3D views, SorNT and SmeNT were classified in three types according to Orhan [9]: A - SmeNT smaller compared to SorNT, B - SmeNT and SorNT of similar size, and $\mathrm{C}-\mathrm{SmeNT}$ greater than SorNT (Fig. 2A-C).

Two observers evaluated each CT examination. In case of disagreement a third examiner was asked to assess the examination, and the case was discussed until consensus was reached.

\section{RESULTS}

Basing on 3D lateral views and MPR, SmeNT was identified in 77 patients; in 59 subjects on both sides, and in 18 unilaterally ( 12 on the right, and 6 on the left). In 8 patients 3D lateral view suggested the presence of SmeNT, but MPR analysis showed anatomical variations that could not be assigned as SmeNT. In case of absence of SmeNT, SO was always located medial to the posteroinferior attachment of SorNT. When SmeNT was present, SO was always located medial to its posteroinferior attachment.

\section{Types of SmeNT}

Two main types of SmeNT were defined basing on coronal plane images in MPR (Fig. 1B): 


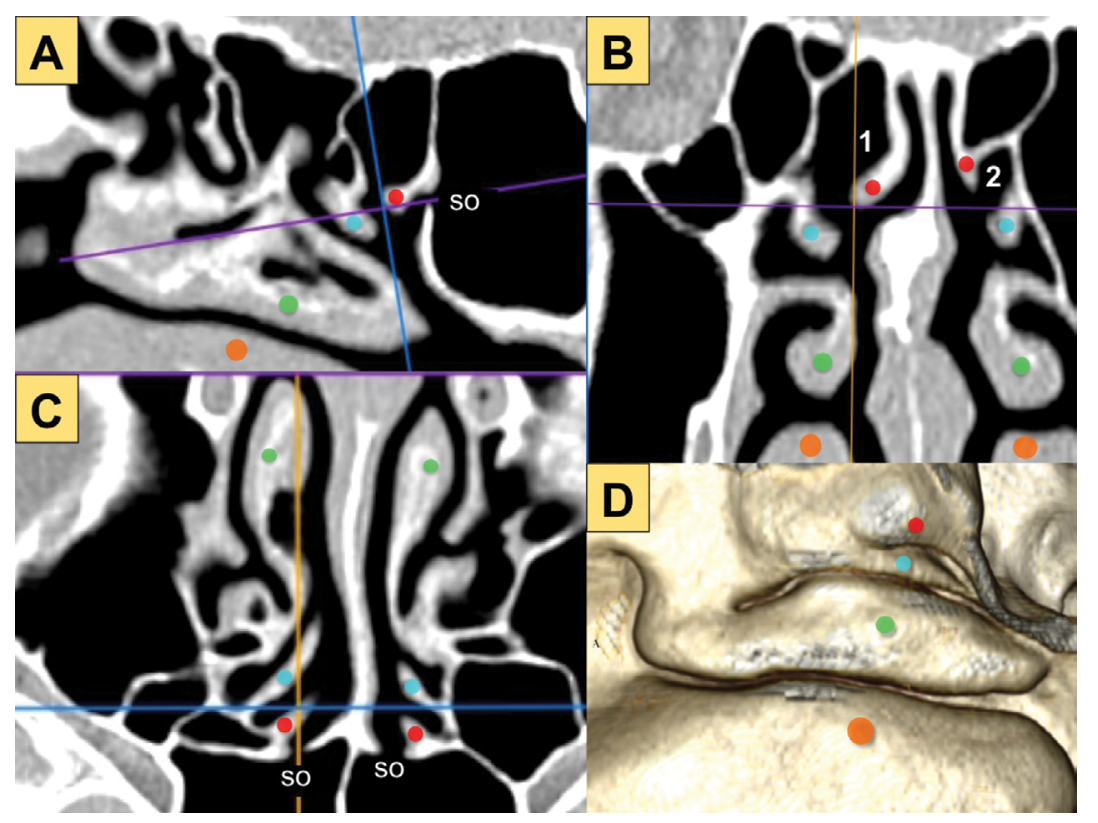

Figure 1. Multiplanar reconstruction of computed tomography; A. Sagittal section; B. Coronal section; C. Axial section; D. Lateral view of a three-dimensional reconstruction of the lateral wall of the nasal cavity; the inferior turbinate — orange dot, the middle turbinate - green dot; superior nasal turbinate (SorNT) — blue dot; supreme nasal turbinate (SmeNT) — red dot; 1 — type 1 SmeNT; 2 - type 2 SmeNT; SO — sphenoid ostium.

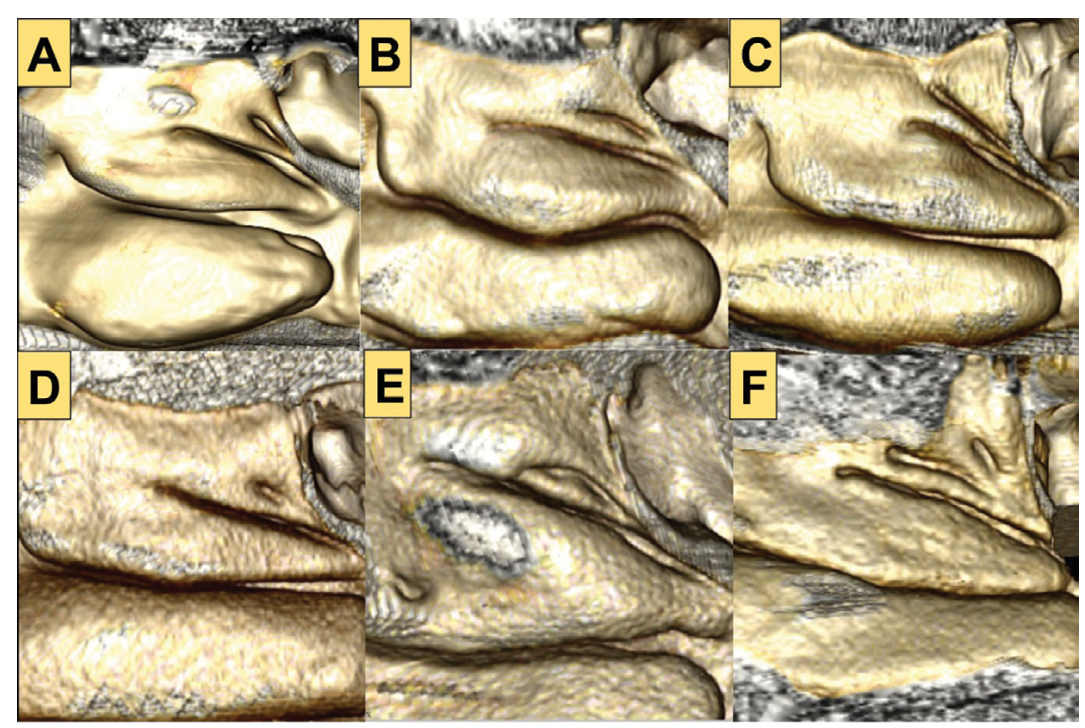

Figure 2. Lateral view of three-dimensional reconstruction of the lateral wall of the nasal cavity; A, B, C. Types of supreme nasal turbinate (SmeNT) according to Orhan; A. SmeNT smaller compared to superior nasal turbinate (SorNT); B. SmeNT and SorNT of similar size; C. SmeNT greater than SorNT; D. Presence of a small oval niche in SorNT with the entrance to the posterior ethmoidal cell; E. SmeNT and SorNT branching off form common trunk arising from the middle turbinate; F. Five nasal turbinates.

1 - forming the ostium to the single posterior ethmoidal cell adjacent to the skull base and/ /or adjacent to the orbit;

2 - forming the entrance to the shallow cavity not adjacent to the skull base or appearing as a fold of the medial wall of the nasal cavity.
The prevalence of the types of SmeNT is presented in Tables 1 and 2.

Six out of 91 type 1 SmeNTs were not adjacent to the skull base, but only to the lamina papyracea. In 8 sides posterior ethmoidal cell drained to the sphenoethmoidal recess through a longitudinal or 
Table 1. Prevalence of different types of supreme nasal turbinate as assessed in multiplanar reconstruction

\begin{tabular}{lccc}
\hline Type & Right (No.) & Left (No.) & Right + left (No.) \\
\hline 1 & 51 & 40 & 91 \\
2 & 20 & 25 & 45 \\
Total & 71 & 65 & 136 \\
\hline
\end{tabular}

Table 2. Prevalence of different types of supreme nasal turbinate as assessed according to Orhan

\begin{tabular}{lccc}
\hline Type & Right (No.) & Left (No.) & Right + left (No.) \\
\hline A & 11 & 10 & 21 \\
B & 38 & 35 & 73 \\
C & 22 & 20 & 42 \\
Total & 71 & 65 & 136 \\
\hline
\end{tabular}

oval funnel located posterior to SorNT, in absence of SmeNT (Fig. 2D).

Sphenoethmoidal (Onodi) cell drained to supreme meatus in 41 subjects (54 sides), and to superior meatus in 37 subjects ( 49 sides).

Nineteen out of 136 SmeNTs were small, hidden and bent laterally in a concave space of the sphenoethmoidal recess, forming a pronounced fold rather than a structure similar to middle or superior turbinates.

\section{Rare abnormalities}

Rare abnormalities observed in MPR and/or 3D lateral views (Fig. 2):

- SmeNT and SorNT branched off from the common stem arising from the middle turbinate - in 8 subjects (Fig. 2E);

- pneumatisation of SmeNT - unilaterally in 7 patients, and bilaterally in 1 patient;

- 5 nasal turbinates (Fig. 2F);

- bifurcated SorNT;

- entrance to posterior ethmoidal cell or shallow cavity anterior and lateral to SO, posterior to SmeNT.

\section{DISCUSSION}

The pathogenesis of chronic rhinosinusitis is multifactorial and still not clear. There is no causative treatment. There has been ongoing discussion in the literature on the extent of surgery needed to control the disease. It is rather advocated to tailor the surgery to the extent of inflammatory changes [4]. Drainage of the posterior ethmoidal cell into the supreme meatus is present in $58 \%$ of subjects from our study at least on one side. If all the posterior ethmoidal cells drain into the superior meatus (in absence of SmeNT or in type 2 SmeNT), perforating the basal lamella of the middle turbinate widens their drainage pathway (Fig. 3B). However, in type 1 SmeNT this manoeuvre will be inadequate to provide sufficient aeration and delivery of saline and topical steroids to the most posterior ethmoidal cell (Fig. 3A). The existence of two separate drainage pathways of the posterior ethmoidal cells in patients with type 1 SmeNT may imply the need for a more extensive surgical approach.

The clinical importance of different types of drainage of sphenoethmoidal (Onodi) cells is unclear. Different types of drainage may suggest the heterogeneous developmental origin of these cells.

The SmeNT is present in $77 \%$ of patients as shown in our study. Stammberger [11] described the appearance of SmeNT over 20 years ago in a handbook of endoscopic surgery. On the other hand SmeNT is rarely seen intraoperatively and there are sparse reports on the use of this structure as a landmark for sphenoid sinus surgery in literature [2]. The possible explanation for this discrepancy is that although SmeNT and SorNT are often of comparable size on the lateral 3D view [9], SmeNT is hidden behind SorNT on anterior view in the majority of cases.

During transnasal approach to the sphenoid sinus, the most important anatomical landmarks are the SO, SorNT, choana and the junction between the nasal septum and anterior face of the sphenoid sinus [10]. These structures are visualised from a distance to allow manipulation with suction or a surgical tool in a narrow field. Under these conditions SmeNT, which is located behind SorNT, in a narrow cleft, is not visible or only partially visible, especially when a $0^{\circ}$ scope is used. Even if both SorNT and SmeNT appear in the field, the groove separating them is not well exposed, and the impression of one irregular surface is created. Only in rare cases do anatomical conditions allow to visualise SmeNT [2]. Visualisation of SmeNT during transnasal approach can be improved with angled scope turned laterally (Fig. 4C).

Transethmoidal approach to the sphenoid sinus using SorNT as a landmark is a well-established method $[1,10]$. After perforating the basal lamella of the middle turbinate, the endoscope is directed slightly towards the midline, from lateral to medial. At this stage of dissection the most superior and posterior aspect of the sphenoethmoidal recess is completely hidden behind SorNT. 


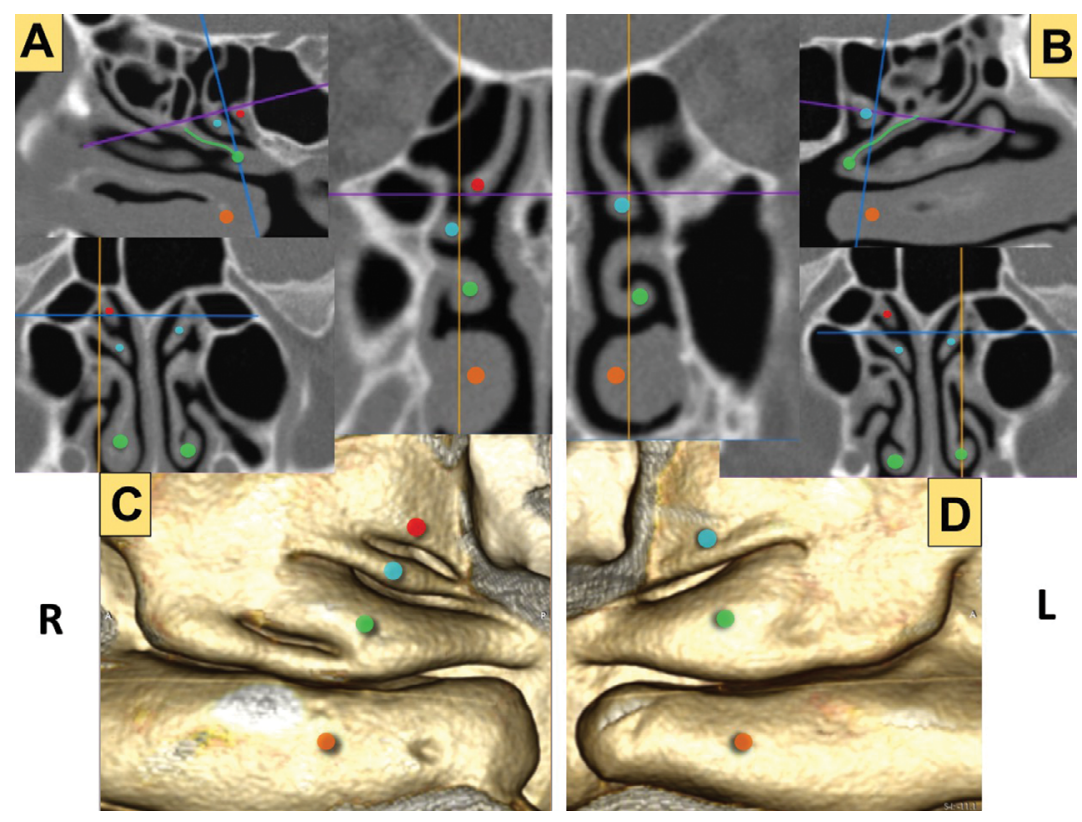

Figure 3. Patient with supreme nasal turbinate (SmeNT) on the right side; A, B. Multiplanar reconstruction; C, D. A lateral view of the lateral wall of the nasal cavity; the inferior turbinate — orange dot, the middle turbinate — green dot, superior nasal turbinate (SorNT) — blue dot, SmeNT — red dot, lower part of the basal lamella of the middle turbinate — green line; $R$ — right side; $L$ - left side. The entrance to the most posterior ethmoidal cell is at the intersection of reference lines. Note that perforation of the basal lamella of the middle turbinate creates a wide pathway to the posterior ethmoidal cells in the absence of the SmeNT (left side).

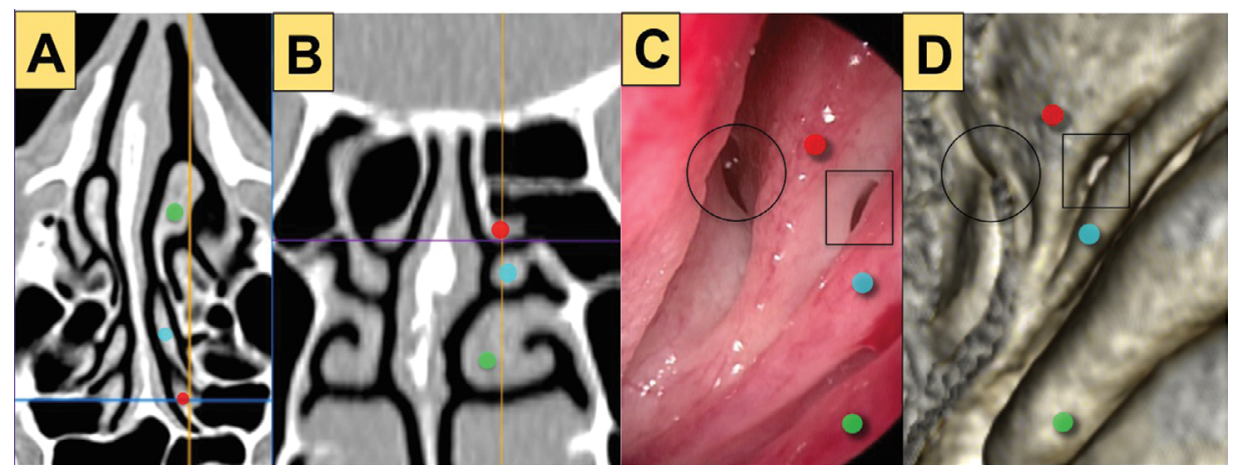

Figure 4. A, B. Coronal and axial computed tomography (CT) reconstruction showing supreme nasal turbinate (SmeNT), superior nasal turbinate (SorNT), sphenoid ostium and the middle turbinate; C. Examination of the left sphenoethmoidal recess with a $30^{\circ}$ scope turned about $45^{\circ}$ laterally, D. Three-dimensional reconstruction of CT of sphenoethmoidal recess. The anterior wall of the sphenoidal sinus was partially removed; A-D — the middle turbinate — green dot; SorNT — blue dot; SmeNT — red dot; C-D — the sphenoid ostium — marked with a circle, the entrance to the most posterior ethmoidal cell — marked with a square.

According to Millar and Orlandi [8], after removal of the lower part of SorNT, SO is identified typically between vertical folds of mucosa. Possibly one of these folds is the lowest part of SmeNT. The most reasonable explanation for why we don't see SmeNT during sphenoethmoidectomy is that when looked at from opened posterior ethmoids, it is not perceived as a turbinate.
In a cadaveric study of 100 heads Kim et al. [6] found that SO is located medial to the inferior part of SorNT in $83 \%$ of cases and in $17 \%$ lateral to this structure. Subsequent studies showed that SO is located medial to SorNT in $98-100 \%[3,8]$. The middle and superior turbinates share structural similarities. It can be approximated that the anterior portion of 
these structures is oriented vertically, their middle segment is positioned in the frontal plane, and the most posterior and lateral part in the axial plane. Thus SO can be located lateral to anterior portion of SorNT and medial to its posterior attachment. Removal of the mucosa from the anterior wall of the sphenoid sinus with posterior part of SorNT may give the impression that SO is located lateral to SorNT [8].

Hypothesis of partial removal of the mucosa from the anterior wall of the sphenoid sinus would also explain differences in the prevalence of SmeNT between the cadaveric studies. Kim et al. [6] did not address the issue of SmeNT; Millar and Orlandi [8] found SmeNT in $8 \%$ of the sides, Orhan et al. [9] in $35 \%$, while Kim et al. [7], who additionally used CT, found it in $50 \%$ of 100 examined sides. In the latter study sagittal scans at $1 \mathrm{~mm}$ intervals without reconstruction were used. Higher prevalence of SmeNT in our study can be explained by the use of thin slice CT together with MPR and 3D reconstruction. Additionally, the planes of reconstruction were aligned for the maximum exposure of evaluated anatomical structures. The above-mentioned cadaveric studies showing the lowest prevalence of SmeNT were performed on a limited number of half-heads (Millar - 47, Orhan -20) compared to 200 sides in our study. Lastly, the prevalence of SmeNT in our study is expressed per individual. When counted per side, it drops to $68 \%$.

It is possible that the high prevalence of SmeNT in our study results from the engorgement of the venous plexuses that would not be seen in cadaver studies or in decongested patients during surgery. The SmeNT is the smallest of all turbinates. It was difficult to set a clear boundary between a mucosal fold and the presence of SmeNT in 6 cases (Fig. 2D).

The exclusion of 1750 CTs from the initial 1850 could have influenced the results. The main reason for exclusion was slice thickness of more than $0.67 \mathrm{~mm}$, as this makes adequate MPR and $3 \mathrm{D}$ reconstruction impossible. This is the least likely cause of bias in our opinion.

However, CT examinations were performed due to sinonasal complaints, and the patients with opacification of the posterior ethmoids, sphenoid sinus and ostium were excluded. This could cause overrepresentation of patients with recurrent episodes of acute rhinosinusitis. Certain anatomical variations are known to predispose to acute rhinosinusitis [4]. It can be hypothesized that some of these variations are associated with the presence of SmeNT, however there is no data proving it.
There is little data in the literature on the relation between SO and SmeNT. Millar and Orlandi [8] found SO medial to SmeNT in all cases. Kim et al. [7] did not describe the site of SO. Orhan et al. [9] did not clearly describe whether SO was medial or lateral to SmeNT.

\section{CONCLUSIONS}

The SmeNT is more common than thought but due to its posterior and superior location to SorNT it is rarely seen intraoperatively. However, if SmeNT is visualised, the SO should be looked for medial to the posteroinferior attachment of this structure, behind its vertical part. Patients with divergent drainage of the posterior ethmoids (with posterior ethmoidal cell draining under SmeNT) may require more extensive surgery to avoid persistence or recurrence of inflammatory disease.

\section{REFERENCES}

1. Bolger WE, Keyes AS, Lanza DC. Use of the superior meatus and superior turbinate in the endoscopic approach to the sphenoid sinus. Otolaryngol Head Neck Surg. 1999; 120(3): 308-313, doi: 10.1016/S0194-5998(99)70267-6, indexed in Pubmed: 10064630.

2. Christmas DA, Mirante JP, Yanagisawa E. Supreme nasal turbinate as a landmark during endoscopic sphenoid sinus surgery. Ear Nose Throat J. 2004; 83(2): 84-85, indexed in Pubmed: 15008437.

3. Eweiss AZ, Ibrahim AA, Khalil HS. The safe gate to the posterior paranasal sinuses: reassessing the role of the superior turbinate. Eur Arch Otorhinolaryngol. 2012; 269(5): 1451-1456, doi: 10.1007/s00405-011-1832-3, indexed in Pubmed: 22086607.

4. Fokkens WJ, Lund VJ, Mullol J, et al. EPOS 2012: European position paper on rhinosinusitis and nasal polyps 2012. A summary for otorhinolaryngologists. Rhinology. 2012; 50(1): 1-12, doi: 10.4193/Rhino50E2, indexed in Pubmed: 22469599.

5. Hopkins C. Nose, Nasal Cavity and Paranasal Sinuses. In: Susan Standring ed. Gray's Anatomy. 561. The Anatomical Basis and Clinical Practice. Forty-first edition, Elsevier 2016.

6. Kim HU, Kim SS, Kang SS, et al. Surgical anatomy of the natural ostium of the sphenoid sinus. Laryngoscope. 2001; 111(9): 1599-1602, doi: 10.1097/00005537-200109000-00020, indexed in Pubmed: 11568612.

7. Kim SS, Lee JG, Kim KS, et al. Computed tomographic and anatomical analysis of the basal lamellas in the ethmoid sinus. Laryngoscope. 2001 ; 111(3): 424-429, doi: 10.1097/00005537200103000-00009, indexed in Pubmed: 11224770.

8. Millar DA, Orlandi RR. The sphenoid sinus natural ostium is consistently medial to the superior turbinate. Am J Rhinol. 2006; 20(2): 180-181, indexed in Pubmed: 16686384.

9. Orhan M, Govsa F, Saylam C. A surgical view of the superior nasal turbinate: anatomical study. Eur Arch Otorhinolaryngol. 2010; 267(6): 909-916, doi: 10.1007/s00405-009-1169-3, indexed in Pubmed: 20012078.

10. Orlandi RR, Lanza DC, Bolger WE, et al. The forgotten turbinate: the role of the superior turbinate in endoscopic sinus surgery. Am J Rhinol. 1999; 13(4): 251-259, indexed in Pubmed: 10485010.

11. Stammberger H. Functional Endoscopic Sinus Surgery. The Messerklinger technique 1991. 